\title{
How the Brain Process Stimulus-Response Conflict? New Insights from Lateralized Readiness Potentials Scalp Topography and Reaction Times
}

\author{
Marc E. Lavoie ${ }^{1,2,3}$, Johannes E. A. Stauder ${ }^{4}$ \\ ${ }^{1}$ Laboratoire de Psychophysiologie Cognitive et Sociale \\ ${ }^{2}$ Centre de Recherche Fernand-Seguin, de l'Hôpital Louis-H. Lafontaine, Montréal, Canada \\ ${ }^{3}$ Département de Psychiatrie, Université de Montréal, Montréal, Canada \\ ${ }^{4}$ Cognitive Neuroscience, Faculty of Psychology and Neuroscience, Maastricht University, Maastricht, The Netherlands \\ Email: marc.lavoie@umontreal.ca
}

Received December 14, 2012; revised January 15, 2013; accepted January 22, 2013

\begin{abstract}
Stimulus-Response Compatibility (SRC) refers to the fact that some tasks are performed easier and better than others because of the way stimuli and responses are paired with each other. To assess the brain responses to stimulus-response conflicts, we investigated the behavioral (accuracy and Reaction Times: RTs) as well as the physiological response (Lateralized Readiness Potentials: LRP) modulations in a positional blocked and a conditional mixed design in twelve university students. Results revealed that the performance was less accurate and the RTs, as well as the LRP onset, were delayed under the mixed conditional design. A greater compatibility effect was also noted on accuracy, RTs and LRP onset latency in the mixed design. Consistent with these findings, smaller peak activation at fronto-central areas suggests that more selective inhibition is needed in a mixed design context. Despite a smaller activation, the topographical distribution is similar in both designs. These results indicate that the translation time between stimulus- and response codes are greater under the mixed instruction, while the similar LRP topography suggests that common neural structures underlie LRPs in response to both type of designs.
\end{abstract}

Keywords: Lateralized Readiness Potentials; Mixed-Blocked Designs; Stimulus Response-Compatibility; Reaction Times

\section{Introduction}

Stimulus-Response Compatibility (SRC) refers to the fact that some tasks are performed easier and better than others because of the way stimuli and responses are paired with each other [1]. SRC are not restricted to particular stimuli, modality or task, indicating the fundamental nature of their underlying cognitive processes. In general terms, two main theoretical views, the serial and the dualtask automatic processing approach, attempted to explain the mechanisms underlying stimulus response conflict. Early SRC research papers [2,3], as well as recent ones [4], proposed that incompatible mapping is slower than compatible mapping because of increased translation time between the stimulus and response code. Accordingly, that coding process arises in series after stimulus identification, but before motor initiation. An alternative explanation is provided by the dual-processing accounts, which attribute SRC effects to the automatic activation of responses [5]. At difference with the classical serial model, automatic activation is not dependent on the effi- ciency of coding processes, but is induced by a separate parallel route in which the response are activated directly and have to be inhibited in case of an incompatible mapping [6]. Both SRC models were elaborated on the basis of overt responses without direct inferences on brain processes underlying stimulus-response conflict.

A considerable number of metabolic brain studies addressed movement related metabolic activity, although none specifically addressed SRC. Increased regional blood flow to hand and finger movements were observed in the contralateral sensorimotor cortex [7,8], premotor cortex, primary motor cortex, anterior cingulate cortex, parietal cortex, the supplementary motor area (SMA) and the prefrontal cortex $[9,10]$. Unfortunately, is the exact functional role of most of these areas is still unclear. Increased blood flow in the Supplementary Motor Area was observed to movements triggered by a stimulus as compared to self paced movements [8] suggesting the SMA to be task related to the contingency of the cue triggering the movement. The anterior cingulate cortex 
was associated response selection [9] and the modulation of commands coming from different regions (e.g. prefrontal) to the motor system [11]. The anterior cingulate cortex was mainly activated when the subjects are forced to choose from a set of competing responses rather than relying on well-established S-R associations. Although these findings might suggest potential mechanisms implicated in SRC, a satisfying understanding of the structures underlying SRC awaits a metabolic study with tasks that specifically modulate stimulus response compatibility. However, even in case a metabolic study isolates the brain structures, involved in the compatibility effect [12], we still ignore the time course of activation, since none of the metabolic techniques offers a high enough time resolution to tear these processes apart. A solution to this dilemma is a topographical Lateralized Readiness Potential (LRP) investigation, which offers a similar time resolution than a single lead LRP but also shows the distribution of the LRP across the scalp at each point in time $[13,14]$. The latter may not only help in determining whether there are qualitative differences in activation between different task manipulations but may also inform us about the underlying brain sources implied in generating the LRP.

We propose to investigate SRC interference through electrophysiological measures of motor activation, the so-called LRPs, that reflects only the lateralized part of the readiness potential, leaving aside electrical potentials associated with non-motor activity [13]. LRPs reveal initial activation of the incorrect response (negative deflection) followed by a delayed activation of the correct response (positive deflection) in case of incompatible response [15]. The concept of automatic activation found considerable support by psychophysiological studies using LRP as an index of selective response preparation.

In order to further examine these hypotheses within a psychophysiological setting, we compared one blocked design, where arrows were presented in two separate blocks of compatible and incompatible sequences and one mixed design where stimulus-response configurations are presented in mixed order and are dependant on the color of the arrow. By means of comparing a mixed with a blocked design, our primary aim is to assess the occurrence of automaticity in SRC interference. Thus, if $\mathrm{SRC}$ is best explained by a dual-task automatic processing model, we predict that interference will be equally present in both the blocked and mixed design (compatibility main effect). On the other hand, if SRC is best explained by a serial processing model, the interference will be more pronounced in the mixed than in the blocked design (design $\mathrm{x}$ compatibility interaction). Although these findings might suggest potential mechanisms implicated in SRC, an understanding of cerebral structures underlying SRC awaits a topographical approach that offers a similar time resolution than a single lead LRP, but with a distribution across the scalp. Hence, our second aim is to investigate the topographical distribution of the LRP to determine whether there are qualitative differences of activation, which will inform us on how the underlying neural structures are involved in stimulus-response conflict, across designs and compatibility conditions (design by electrodes or a design by compatibility by electrodes).

\section{Methodology}

\subsection{Participants}

The participants were 8 female and 4 male students from the University of Montreal. They were right handed, and had normal color vision and normal or corrected to distant vision. Their average chronological age was 23.8 years $(\mathrm{sd}=3.4)$ and the intelligence level was determined by the Raven's Advanced Progressive Matrices. The scores on this test were $10.8(\mathrm{sd}=1.5)$ for Set I $(12 / 12)$ and $25.5,(\mathrm{sd}=5.9)$ for Set II $(36 / 36)$. The total average raw scores $(48 / 48)$ was 38.3 , sd $=7.0$. Handedness was determined by the Edinburgh Inventory (Old-field, 1971) on which the participants showed an average score of $85.6, \mathrm{sd}=27.8$ (range -100 to 100 ). The color vision was determined by means of the Dvorine Pseudo-Isochromatic Plates (2ed, 1953) on which the participants correctly identified $97 \%$ of the 15 plates correct $(\mathrm{sd}=0.05)$. None of the participants had a score below the normal color vision threshold of $89.5 \%$ (2 unidentified plates). Letter plates (Home vision care, Anaheim California), determined distance vision on which all subjects showed $100 \%$ correct letter identification above the $20 / 40$ level. The Table 1 shows the absence of sex differences on all these variables.

Table 1. Demographic and psychological variables.

\begin{tabular}{cccc}
\hline & Female & Male & \\
\hline Age (years) & Mean (SD) & Mean (SD) & t-test \\
Laterality (\% right) & $82.5(29)$ & $82.7(27)$ & $\mathrm{ns}$ \\
Schooling (years) & $17.1(2)$ & $16.5(1)$ & $\mathrm{ns}$ \\
Intelligence (raw score) & & & \\
Raven set I & $10.75(2)$ & $11(1)$ & $\mathrm{ns}$ \\
Raven set II & $26.63(7)$ & $29.25(4)$ & $\mathrm{ns}$ \\
Raven-total & $37.38(8)$ & $40.25(6)$ & $\mathrm{ns}$ \\
Color vision (\%) & $98(0.5)$ & $96(0.7)$ & $\mathrm{ns}$ \\
Letter plates (\%) & & & \\
Left eye & 100 & 97 & $\mathrm{~ns}$ \\
Right eye & 100 & 94 & $\mathrm{~ns}$ \\
Both eyes & 100 & 100 & $\mathrm{~ns}$ \\
\hline
\end{tabular}




\subsection{Procedures}

Participants were administered three tasks: 1) In the positional blocked compatible tasks (100 trials), participants were asked to respond with the hand corresponding to the direction of the arrow, while ignoring its color. 2) The positional blocked incompatible (100 trials) required responding with the hand opposite to the direction of the arrow, while ignoring color. 3) The conditional mixed task (200 trials) required a hand response corresponding to the direction of the arrow to one color and with the hand opposite to the direction of the arrow to the other color. The arrows were presented for $350 \mathrm{~ms}$ (Inter-Stimulus-Interval $=2000-2500 \mathrm{~ms}$ ).

The EEG signals were recorded concomitantly and sampled at $250 \mathrm{~Hz}$, with a high- and low-pass filter of 0.01 and $30 \mathrm{~Hz}$ respectively, recorded from 20 tin electrodes mounted in a nylon cap, placed at F7, F8, FC3, FC4, T7, T8, C3, C4, C1, C2, CP3, CP4, TP7, TP8, P7, P8, P3, P4, O1, O2 referenced to linked earlobes (impedance $<5 \mathrm{kOhms}$ ). The EEG was corrected for artifacts with a dynamic regression in the frequency domain [16] and correct trials averaged off-line from stimulus onset.

\subsection{Statistics and Processing}

The stimulus-locked LRP, obtained from subtraction [13], were reduced to 10 locations (F7', F3', FC3', C1', C3', P3', P7', T7', TP7', O1'). The proportional onset was determined as $20 \%$ of the maximum positive peak of the LRP within 50 - $600 \mathrm{~ms}$ post-stimulus. Two factors including DESIGN (mixed vs blocked) and COMPATIBILITY (compatible vs incompatible) were analyzed for Reaction Times (RTs) and accuracy separately. Repeated measures factor (ELECTRODES) was incorporated in the analysis with LRP onset and peak amplitude.

\section{Results}

\subsection{Performances}

Accuracy was significantly better to the blocked (2\% error rate) than to the mixed ( $8 \%$ error rate) design $(\mathrm{F} 1,11)=$ 18.33, $\mathrm{p}<0.001)$. Consistently, RTs were significantly faster in the blocked (372 ms) than in the mixed (561 ms) design $(\mathrm{F}(1,11)=255.83, \mathrm{p}<0.001)$. In both blocked and mixed design (Figure 1), the compatible yielded faster RTs than the incompatible condition $(\mathrm{F}(1,11)=15.44, \mathrm{p}$ $<0.005)$, but that incompatibility effect was only significant in the blocked design $(\mathrm{t}(11)=-5.12, \mathrm{p}<0.001)$.

\subsection{LRP Onset Latency}

The LRP onset latency revealed that there were main effects of design $(F(1,11)=48.02, \mathrm{p}<0.001)$ and compatibility $(\mathrm{F}(1,11)=5.88, \mathrm{p}<0.05)$. The LRP onset was longer in the mixed (290 ms) than in the blocked (196 ms) design and the compatible $(218 \mathrm{~ms})$ yielded faster latency than the incompatible $(268 \mathrm{~ms})$. In addition, there was a design by compatibility interaction $(\mathrm{F}(1,11)=8.78$, $\mathrm{p}<0.05)$ revealing that the LRP latency difference between compatible and incompatible conditions was larger in the mixed $(90 \mathrm{~ms})$ than in the blocked (4 ms) design (Figure 2). The LRP waveforms (Figure 3) revealed an incorrect activation (negative deflection) peaking at 250 $\mathrm{ms}$ in the mixed design only. This was followed by a correct activation (positive deflection) peaking at approximately $300 \mathrm{~ms}$ in response to the blocked design and at $500 \mathrm{~ms}$ post-stimulus in the mixed design.

\subsection{LRP Peak Amplitude}

The results of the LRP peak amplitude activation revealed that there was main effects of design $(\mathrm{F}(1,11)=$ $22.87, \mathrm{p}<0.001)$ and compatibility $(\mathrm{F}(1,11)=4.90, \mathrm{p}<$ 0.05). The LRP amplitude was smaller in the mixed (3.1 $\mathrm{uV})$ than in the blocked $(4.3 \mathrm{uV})$ design and the incompatible $(3.5 \mathrm{uV})$ yielded smaller amplitude than the com-

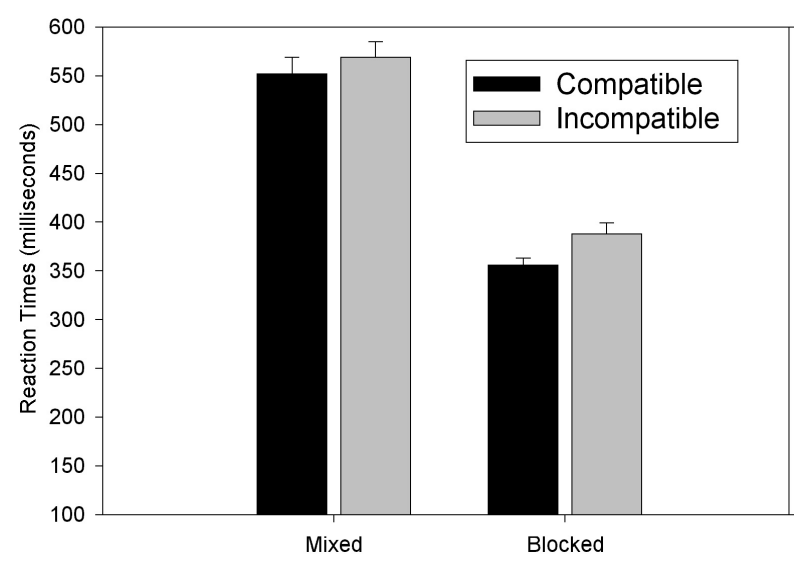

Figure 1. Reaction times in function of compatibility, in comparing blocked and mixed designs.

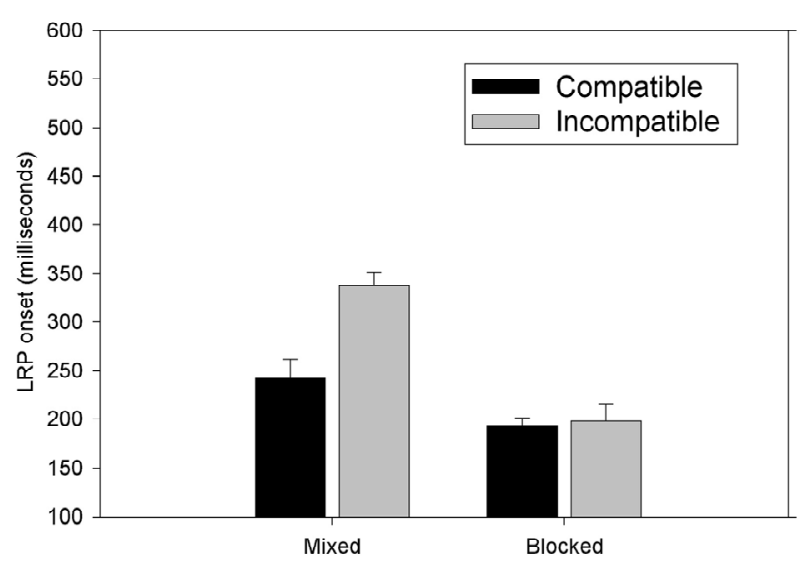

Figure 2. Comparison of the SRC compatibility effect from the LRP onset latency, related to the correct activation in response to the mixed and blocked design. 

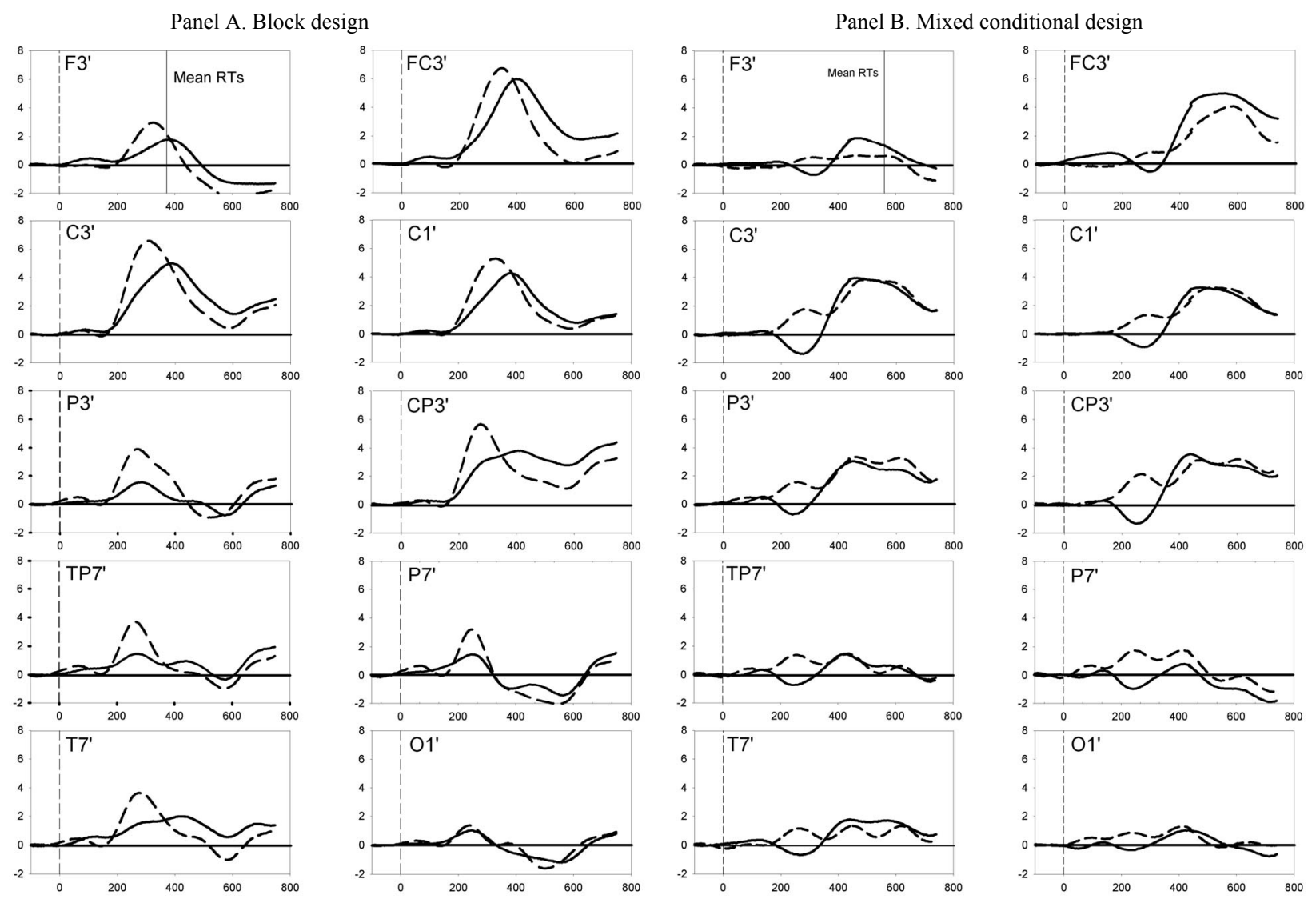

Figure 3. Scalp distribution of the stimulus-locked LRP compatibility effect related to the blocked (panel A) and mixed design (panel B). For both panels, the solid bold line shows the LRPs to the incompatible, while the dashed line shows the LRPs in response to the compatible. The $y$-axis denotes the amplitude in microvolts, while the $x$-axis denotes the time scale in milliseconds. The vertical dashed lines refer to the stimulus onset, while the solid vertical line shows the mean reaction times (RTs) for each task. The negative polarity deflections denote the incorrect activation of the response while the positive deflections indicate the correct activation of the response.

patible $(4 \mathrm{uV})$. Finally, a design by electrode interaction was also found on the LRP peak amplitude $(\mathrm{F}(3.98,43.83)$ $=3.74, \mathrm{p}<0.05)$ showing that the LRP amplitude was larger in the blocked than in the mixed design at FC3', C3'and CP3' respectively (see Figure 4).

\section{Discussion}

Our findings consistently showed that performance was less accurate and the RTs, as well as LRP onset, were delayed under the mixed conditional design as compared with the blocked design. This indicates that the translation time between stimulus and response codes are greater under the mixed condition. Consistent with these findings, smaller peak activation at fronto-central regions suggests that more selective inhibition is solicited, when the program for the correct activation is retrieved in the mixed design context [17]. Single neuron studies also support response competition effects of preliminary visual information in the frontal cortex revealed by the LRPs $[18,19]$. Despite the smaller activation observed in the mixed design, the topographical distribution is similar than in the blocked design, suggesting that common neural structures underlie LRPs in response to both designs.

Additionally, a greater compatibility effect on accuracy, RTs and LRP onset latency in blocked than in mixed design, supports a model of serial activation of the response as it were found in similar SRC studies [20,21]. This suggests that, when stimuli are presented in a blocked design, both compatible and incompatible conditions directly (or automatically) activate the correct response. However, when stimuli are presented in a mixed design an additional step must be performed i.e. the activated response together with its program is first aborted (negative waveform peaking at $300 \mathrm{~ms}$ post-stimulus) in the incompatible condition, and the program for the correct response is retrieved later in time (positive waveform peaking at $500 \mathrm{~ms}$ post-stimulus), favoring a dual route processing explanation of the mixed SRC interference [22]. When the response spatially corresponds to 


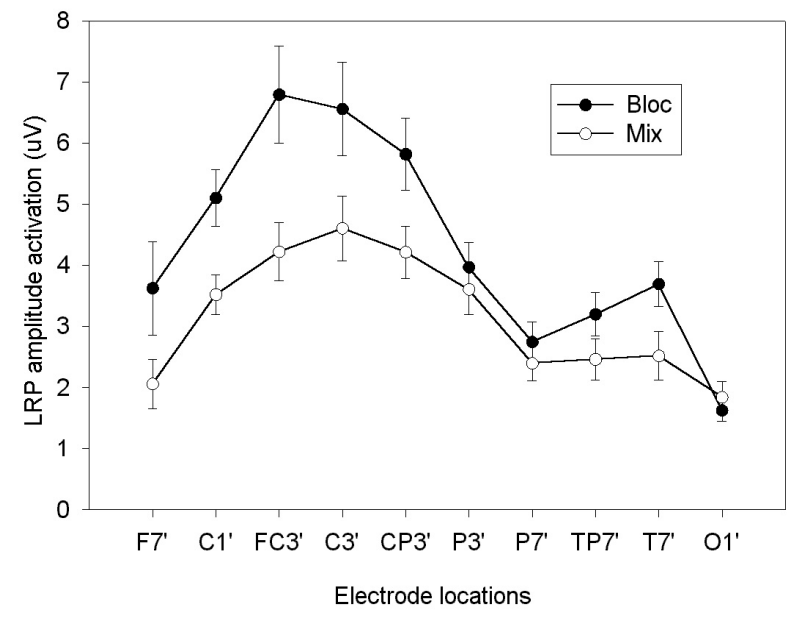

Figure 4. Stimulus-locked LRP scalp distribution from lateralized locations showing the correct maximum activation (with standard error of the mean) to blocked and mixed designs (averaged across compatible-incompatible conditions). The figure show a design by electrode interaction found on the LRP peak amplitude. The LRP amplitude was larger in the blocked than in the mixed design at FC3', C3'and CP3' respectively.

stimulus location a fast direct route is activated, whereas a slower indirect (controlled) route activates the intentionally selected response [23]. However, the discrepancy in the compatibility effect between RT and LRP onset measures in the mixed design could be attributed to the larger LRP-RT difference to the mixed $(271 \mathrm{~ms})$ as compared to the blocked design (176 ms). Thus, in the context of a mixed design, LRP onset is more distant from the RT and produces a delayed speed of response activation as compared to the blocked design because of a delayed translation time to decode the response program.

In conclusion, our data showed a slower LRP onset and reaction times to the incompatible mapping, greater in the mixed design and favor a serial processing explanation of the stimulus-response conflict. Moreover, the LRP amplitude activation during the mixed design consistently showed smaller amplitude at fronto-central areas (design by electrode interaction), revealing that more selective inhibition was necessary to perform the conditional mixed task.

\section{Acknowledgements}

This work was supported by a Fonds pour la Recherche en Santé du Québec (FRSQ) clinical research (5271) and the FRSQ chercheur-boursier awarded to MEL. At last but not the least, we thank all participants for their precious contribution in this study.

\section{REFERENCES}

[1] J. R. Simon, "The Effects of an Irrelevant Directional Cue on Human Information Processing," In: R. W. Proctor, Ed., Stimulus-Response Compatibility: An Integrated Perspective, Elsevier, Amsterdam, 1990, pp. 31-86.

[2] P. M. Fitts and C. M. Seeger, "S-R Compatibility: Spatial Characteristics of Stimulus and Response Codes," Journal of Experimental Psychology, Vol. 46, No. 3, 1953, pp. 199-210. doi:10.1037/h0062827

[3] P. M. Fitts and R. L. Deininger, "S-R Compatibility: Correspondence among Paired Elements within Stimulus and Response Codes," Journal of Experimental Psychology, Vol. 48, No. 6, 1954, pp. 483-492. doi:10.1037/h0054967

[4] B. Hommel, "Attention and Spatial Stimulus Coding in the Simon Task: A Rejoinder to Van der Lubbe and Abrahamse," Acta Psychologica (Amsterdam), Vol. 136, No. 2, 2011, pp. 265-268. doi:10.1016/j.actpsy.2010.10.002

[5] C. D. Wickens, M. Vidulich and D. Sandry-Garza, "Principles of S-C-R Compatibility with Spatial and Verbal Tasks: The Role of Display-Control Location and VoiceInteractive Display-Control Interfacing," Human Factors, Vol. 26, No. 5, 1984, pp. 533-543.

[6] S. Kornblum, T. Hasbroucq and A. Osman, "Dimensional Overlap: Cognitive Basis for Stimulus-Response Compatibility-A Model and Taxonomy," Psychological Review, Vol. 97, No. 2, 1990, pp. 253-270. doi:10.1037/0033-295X.97.2.253

[7] J. Olesen, "Contralateral Focal Increase of Cerebral Blood Flow in Man during Arm Work," Brain, Vol. 94, No. 4, 1971, pp. 635-646. doi:10.1093/brain/94.4.635

[8] P. Remy, et al., "Movement- and Task-Related Activations of Motor Cortical Areas: A Positron Emission Tomographic Study," Annals of Neurology, Vol. 36, No. 1, 1994, pp. 19-26. doi:10.1002/ana.410360107

[9] C. D. Frith, et al., "Willed Action and the Prefrontal Cortex in Man: A Study with PET," Proceedings of Biological sciences, Vol. 244, No. 1311, 1991, pp. 241-246. doi:10.1098/rspb.1991.0077

[10] M. P. Deiber, et al., "Cortical Areas and the Selection of Movement: A Study with Positron Emission Tomography," Experimental Brain Research, Vol. 84, No. 2, 1991, pp. 393-402. doi:10.1007/BF00231461

[11] T. Paus, et al., "Role of the Human Anterior Cingulate Cortex in the Control of Oculomotor, Manual, and Speech Responses: A Positron Emission Tomography Study," Journal of Neurophysiology, Vol. 70, No. 2, 1993, pp. 453469.

[12] B. S. Peterson, et al., "An Event-Related Functional MRI Study Comparing Interference Effects in the Simon and Stroop Tasks," Cognitive Brain Research, Vol. 13, No. 3, 2002, pp. 427-440. doi:10.1016/S0926-6410(02)00054-X

[13] M. G. Coles, "Modern Mind-Brain Reading: Psychophysiology, Physiology, and Cognition," Psychophysiology, Vol. 26, No. 3, 1989, pp. 251-269. doi:10.1111/j.1469-8986.1989.tb01916.x

[14] K. M. Spencer and M. G. Coles, "The Lateralized Readiness Potential: Relationship between Human Data and Response Activation in a Connectionist Model," Psychophysiology, Vol. 36, No. 3, 1999, pp. 364-370. doi:10.1017/S0048577299970749 
[15] M. Eimer, "Stimulus-Response Compatibility and Automatic Response Activation: Evidence from Psychophysiological Studies," Journal of Experimental Psychology: Human Perception \& Performance, Vol. 21, No. 4, 1995, pp. 837-854. doi:10.1037/0096-1523.21.4.837

[16] J. C. Woestenburg, M. N. Verbaten and J. L. Slangen, "The Removal of the Eye-Movement Artifact from the EEG by Regression Analysis in the Frequency Domain," Biological Psychology, Vol. 16, No. 1-2, 1983, pp. 127147. doi:10.1016/0301-0511(83)90059-5

[17] R. de Jong, "Perception-Action Coupling and S-R Compatibility," Acta Psychologica (Amsterdam), Vol. 90, No. $1-3,1995$, pp. 287-299. doi:10.1016/0001-6918(95)00036-T

[18] J. Miller, A. Riehle and J. Requin, "Effects of Preliminary Perceptual Output on Neuronal Activity of the Primary Motor Cortex," Journal of experimental psychology: Human perception \& performance, Vol. 18, No. 4, 1992, pp. 1121-1138. doi:10.1037/0096-1523.18.4.1121

[19] A. P. Georgopoulos, et al., "Mental Rotation of the Neu- ronal Population Vector," Science, Vol. 243, No. 4888, 1989, pp. 234-236. doi:10.1126/science. 2911737

[20] R. W. Proctor, et al., "Dissociation of S-R Compatibility and Simon Effects with Mixed Tasks and Mappings," Journal of Experimental Psychology: Human Perception \& Performance, 2012. doi:10.1037/a0029923

[21] R. W. Proctor and K. P. Vu, "Stimulus-Response Compatibility for Mixed Mappings and Tasks with Unique Responses," Quarterly Journal of Experimental Psychology, Vol. 63, No. 2, 2010, pp. 320-340. doi: $10.1080 / 17470210902925270$

[22] M. Tagliabue, et al., "The Role of Long-Term Memory and Short-Term Memory Links in the Simon Effect," Journal of Experimental Psychology: Human Perception and Performance, Vol. 26, No. 1, 2000, pp. 648-670. doi:10.1037/0096-1523.26.2.648

[23] H. Leuthold, "The Simon Effect in Cognitive Electrophysiology: A Short Review," Acta Psychologica (Amsterdam), Vol. 136, No. 2, 2011, pp. 203-211. doi:10.1016/j.actpsy.2010.08.001 